Société d'histoire de la révolution de 1848 et des

révolutions du XIXe siècle

56 | 2018

Un autre $\mathrm{XIX}^{\mathrm{e}}$ siècle : I'Inde sous domination coloniale

\title{
Se repérer au Tonkin. Usage d'un croquis vietnamien par les Français lors de la conquête,
} 1883-1886

Find your bearings in Tonkin. Use of a Vietnamese sketch by the French during the conquest, 1883-1886

Sich in Tonkin zurechtfinden. Verwenden einer vietnamesischen Skizze durch Franzosen während der Eroberung, 1883-1886

Marie de Rugy

\section{CpenEdition}

Journals

Édition électronique

URL : http://journals.openedition.org/rh19/5613

DOI : 10.4000/rh19.5613

ISSN : $1777-5329$

Éditeur

La Société de 1848

Édition imprimée

Date de publication : 15 octobre 2018

Pagination : 173-185

ISSN : 1265-1354

Référence électronique

Marie de Rugy, «Se repérer au Tonkin. Usage d'un croquis vietnamien par les Français lors de la conquête, 1883-1886 », Revue d'histoire du XIXe siècle [En ligne], 56 | 2018, mis en ligne le 15 octobre 2020, consulté le 05 janvier 2021. URL : http://journals.openedition.org/rh19/5613 ; DOI : https:// doi.org/10.4000/rh19.5613 


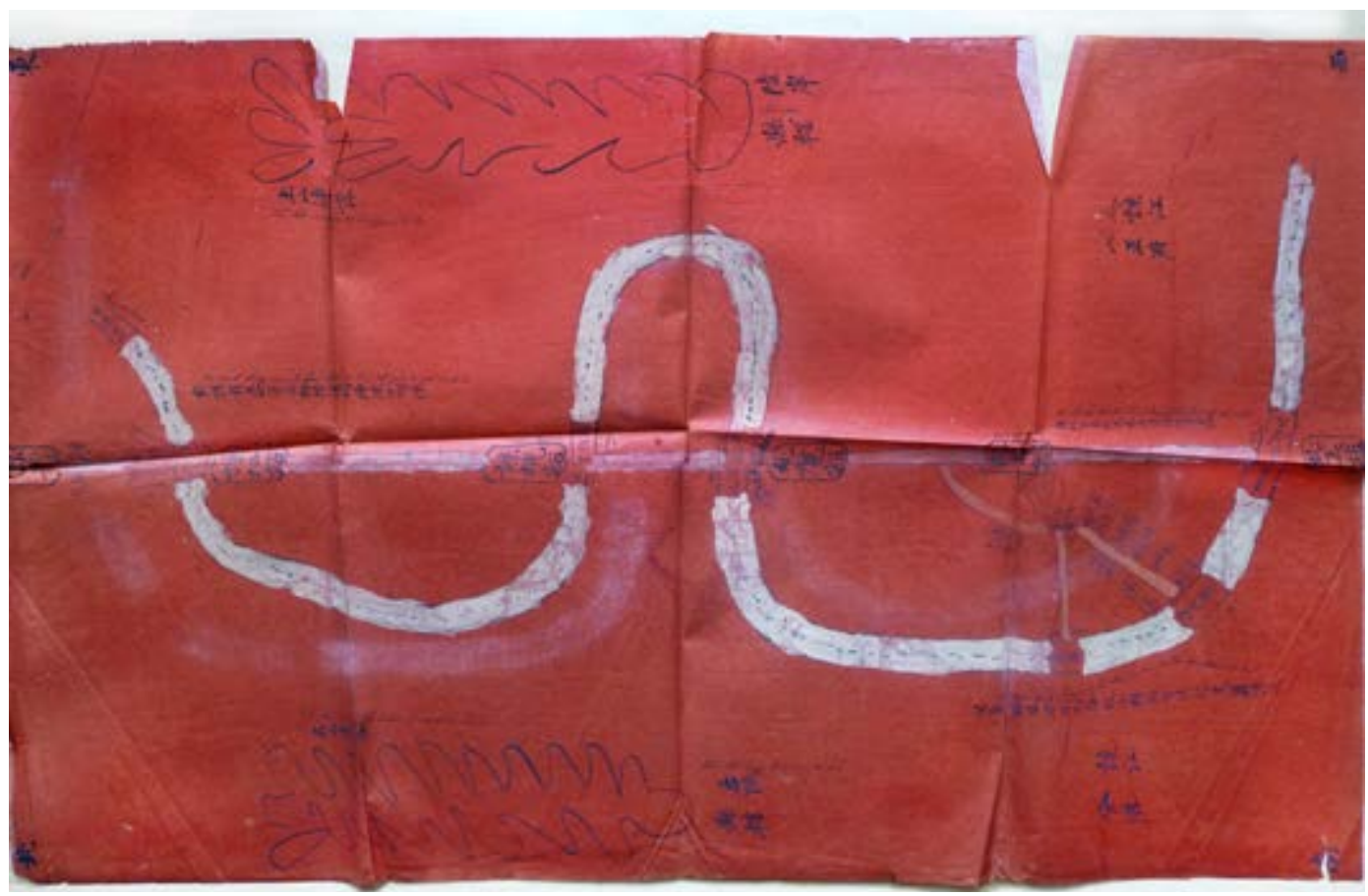

Croquis vietnamien dessiné pendant la conquête du Tonkin, probablement 1884 . Source: SHD, $10 H 6,75 \times 50 \mathrm{~cm}$.

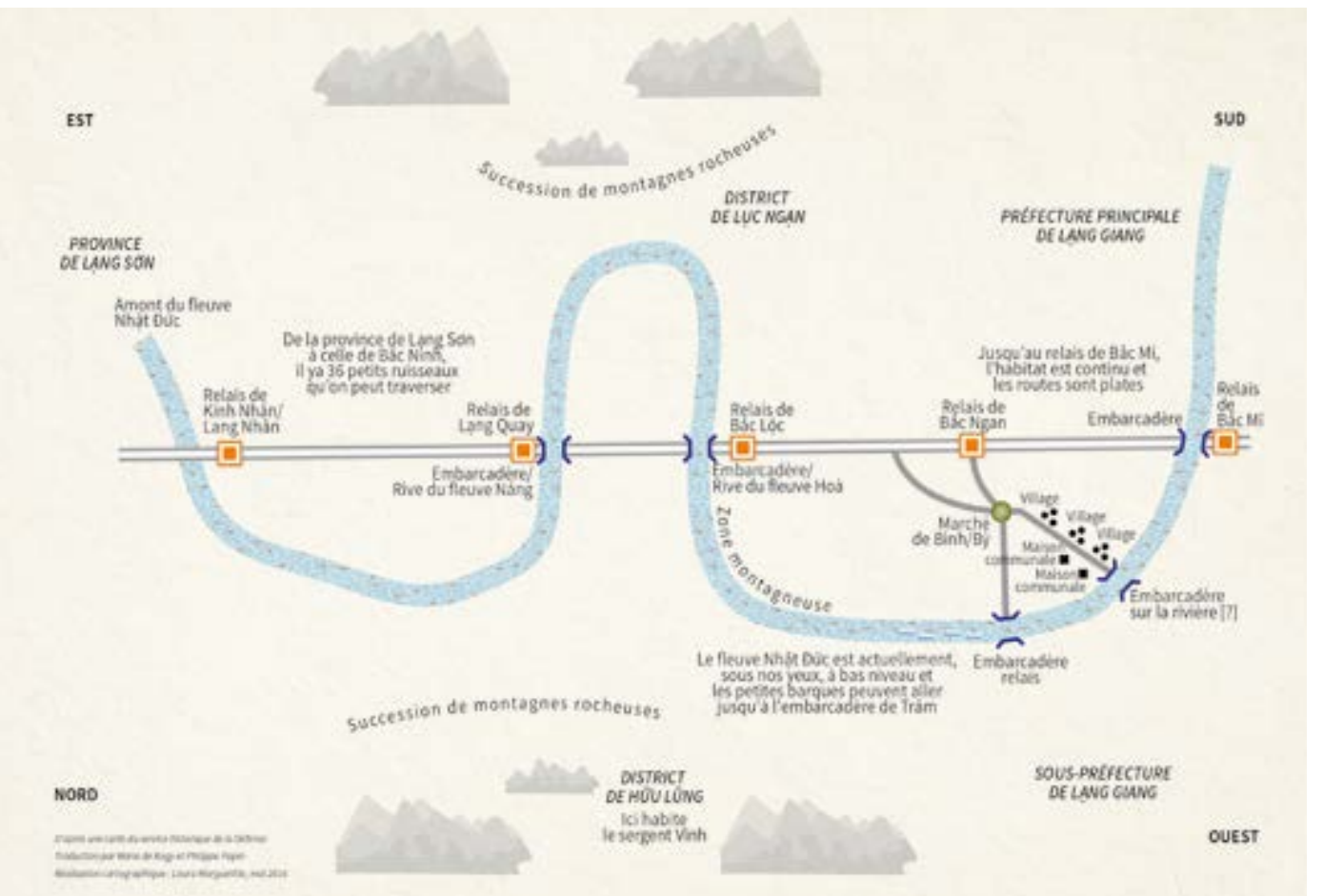

Croquis explicatif, d'après une carte du service historique de la Défense. Traduction par Marie de Rugy et Philippe Papin. Réalisation cartographique : Laura Margueritte, mai 2016. 


\section{MARIE DE RUGY}

\section{Se repérer au Tonkin. Usage d'un croquis vietnamien par les Français lors de la conquête, 1883-1886}

Tracés sur papier ou sur soie, à l'encre ou au crayon, simples croquis griffonnés à la hâte ou travaux plusieurs fois retouchés, les documents cartographiques élaborés par des autochtones à la fin du XIX ${ }^{\mathrm{e}}$ siècle dans la péninsule indochinoise donnent à voir une diversité de formes et de contenus ${ }^{1}$. Ils restent rares dans les archives européennes et asiatiques et l'un d'entre eux, conservé au Service historique de la Défense (SHD) à Vincennes, suscite de nombreuses questions. De taille moyenne $(75 \times 50 \mathrm{~cm})$, peint avec des couleurs végétales sur papier, il est de facture assez simple. Les méandres d'un fleuve représenté en blanc, le Nhật Đức ${ }^{2}$, se dégagent sur le fond rouge et sont traversés par une route rectiligne, plus mince, en blanc également. À l'ouest de la carte, quatre routes de moindre importance sont représentées. Un marché se trouve à leur intersection, tandis que trois villages et deux maisons communales sont mentionnés le long d'un des axes. Cinq toponymes encadrés sont notés à intervalles réguliers, le plus souvent au point de rencontre du fleuve et de la route principale, et six autres sont écrits sur le fleuve lui-même. Les noms des divisions administratives, au nombre de cinq, apparaissent dans les parties plus périphériques du document. Elles nous apprennent que l'espace représenté se situe entre Hanoi et Lạng Sơn, environ à équidistance de chacune des deux villes - une centaine de kilomètres $^{3}$. Deux figurés semblables à des feuilles de chêne symbolisent les zones montagneuses, de part et d'autre des voies de communication. Trois article.

1. Je remercie Philippe Papin pour ses commentaires sur une version préliminaire du présent

2. Aussi appelé Sông Thương, ce fleuve, long de 157 kilomètres traverse les provinces de Lạng Sơn, Bắc Giang et Hải Dương.

3. Les districts de Hữu Lũng au nord-ouest du croquis et Lục Ngạn au sud-est appartiennent alors à la sous-préfecture de Lạng Giang, mentionnée à l'ouest, qui dépend de la province de Bắc Ninh. $C f$. Ngô Đức Thọ, Philippe Papin et Văn Nguyên Nguyễn [dir.], Đông Khánh dịa dre chi, Hanoi, Institut Hán Nôm, EFEO, 2003, p. 527 et $s q$. Le territoire représenté se trouve à la frontière des provinces actuelles de Bắc Giang et Lạng Sơn. 
commentaires textuels, le long des axes fluvial ou terrestre, sont écrits en caractères chinois et transcrits en vietnamien romanisé, avec de nombreuses fautes ${ }^{4}$. Le document ne possède ni titre, ni légende, ni auteur, ni date. Il est malaisé - et probablement vain - de vouloir le faire entrer dans une catégorie géographique occidentale. Pour le terrain indochinois, dans les sources françaises de la fin du XIX ${ }^{e}$ siècle, le terme de "carte» est le plus souvent utilisé de manière indifférenciée pour les cartes topographiques comme pour les documents géographiques autochtones trouvés sur le terrain ou commandés à des habitants. On trouve aussi le mot "croquis", que l'on préferera ici : il renvoie à un dessin rapide, effectué à main levée et dégageant à grands traits l'essentiel du sujet, l'objectif n'étant pas de tout localiser, mais de sélectionner les quelques éléments jugés utiles.

La présence de ce document dans les archives françaises de la conquête du Tonkin (1883-1886) est un indice de taille pour sa datation et le contexte de sa production, même si les circonstances de son acheminement sont inconnues, contrairement à d'autres croquis dont on sait à qui ils ont appartenu'. Les Français occupent en effet le sud de la péninsule indochinoise depuis les années 1860, créant la colonie de Cochinchine et le protectorat du Cambodge $^{6}$. La citadelle de Hanoi est ensuite prise une première fois en 1873, sans que le gouvernement français ne soutienne ce coup de force. En 1883 en revanche, il y a une véritable volonté politique de conquérir le delta du fleuve Rouge, nourrie de l'espoir que le fleuve serve au commerce entre les possessions françaises et la Chine. Au mois d'août 1883, la cour vietnamienne reconnait le protectorat de la France sur l'Annam et le Tonkin ${ }^{7}$ Le traité, signé à Huế, ouvre plusieurs années de conflit entre les militaires français, soutenus par des troupes autochtones, et un ennemi aux facettes et aux motivations multiples: armée régulière chinoise, combattants vietnamiens qui résistent à la présence française, soldats en rupture de ban. La guerre avec la Chine se termine le 9 juin 1885 : par le traité de Tianjin, cette dernière accepte de retirer ses troupes et d'abandonner ses droits sur le royaume d'Annam, tributaire depuis le $\mathrm{X}^{\mathrm{e}}$ siècle, au profit de la France. Les autres ennemis sont plus longs à éradiquer, mais si la "pacification" se prolonge au Tonkin, la conquête est considérée par les autorités françaises comme terminée en 1886.

4. L'écriture vietnamienne romanisée, aussi appelée chũ quốc ngũu, est inventée par le jésuite Alexandre de Rhodes au XVII ${ }^{e}$ siècle. Elle correspond à l'alphabet latin augmenté d'accents diacritiques.

5. Le croquis ne semble pas faire partie d'une collection constituée sur le terrain par un militaire, tels les nombreux documents trouvés dans les papiers du colonel Donnier, décédé le 28 mars 1888, et conservés au Service historique de la Défense (SHD, $10 \mathrm{H} 3$, dossier 9).

6. Pierre Brocheux et Daniel Hémery, Indochine. La colonisation ambigüe (1858-1954), Paris, La Découverte, 1995.

7. Pour un point sur la chronologie de la conquête, $C f$. Charles Fourniau, Vietnam. Domination coloniale et résistance nationale, 1858-1914, Paris, Indes savantes, 2002. 
Le croquis présenté est élaboré dans ce contexte afin de servir, comme d'autres, aux militaires français. Ce type de documents semble en effet spécifique à la période coloniale - on ne dispose d'aucun exemple auparavant et les seules cartes demandées par le pouvoir vietnamien sont des cartes provinciales ou de district pour établir les géographies royales, ce qui n'est pas le cas ici. Nous reprenons à notre compte l'hypothèse formulée par John Whitmore à propos d'une dizaine de cartes manuscrites, principalement des plans de villes vietnamiennes conservés à la Bibliothèque nationale de France et à la Bayerische Staatsbibliothek ${ }^{8}$ : ces documents auraient permis aux Français de se repérer sur le terrain au moment de la conquête, alors qu'ils ne disposaient que de peu de connaissances géographiques précises sur ces territoires. Ces cartes sont cependant en français, dressées à partir de "plans et renseignements annamites", ce qui n'est pas le cas de notre croquis, où le français est absent, contrairement à une autre carte vietnamienne conservée dans le même dossier'. Cela nous conduit donc à l'étude des savoirs autochtones en situation coloniale. Les travaux sur les "savoirs vernaculaires", terme qui renvoie aux connaissances produites à une échelle locale et qui n'ont pas prétention à l'universalité, ont principalement pris pour objet l'Afrique. La réflexion porte en priorité sur l'oralité et conduit à favoriser les enquêtes de terrain dans une approche ethnographique pour accéder aux discours africains $^{10}$, même si cela n'exclut pas quelques travaux sur l'écrit ${ }^{11}$. L'enjeu est notamment de mettre en lumière les logiques de production des savoirs ${ }^{12}$. La quête de renseignements auprès des populations locales, au moment de l'exploration, de la conquête ou de l'administration des territoires, suscite aussi l'intérêt grandissant des historiens parce qu'elle témoigne de la rencontre entre colonisateurs et colonisés, alors que les populations locales ne sont pas toutes acquises à la cause des Français ${ }^{13}$. Ces travaux ont une pertinence pour l'étude de la péninsule indochinoise, mais la variété des productions cartographiques en Asie du Sud-Est constitue une spécificité par rapport au cas africain et mérite des analyses approfondies.

Il convient de comprendre ce qu'est ce document cartographique, qui a pu le produire, dans quel contexte précis, avec quel objectif, et ce qu'il

8. John Whitmore, "Cartography in Vietnam”, in John Brian Harley et David Woodward (eds), History of Cartography, Chicago, University of Chicago Press, 1992, volume 2, book 2, p. 478-508. Cet ouvrage a contribué à renouveler en profondeur une histoire de la cartographie jusqu'alors très positiviste et cantonnée aux pratiques occidentales dans la tradition ptoléméenne.

9. SHD, $10 \mathrm{H6}$, [croquis vietnamien avec annotations françaises], $38 \times 30 \mathrm{~cm}$.

10. Claude-Hélène Perrot, Sources orales de l'histoire de l'Afrique, Paris, Éditions du CNRS, 1989.

11. Camille Lefebvre, Isabelle Surun, «Exploration et transferts de savoir : deux cartes produites par des Africains au début du XIXe siècle», Mappemonde, 92-4, 2008; Éloi Ficquet, Aissatou MbodjPouye, "Cultures de l'écrit en Afrique. Anciens débats, nouveaux objets", Annales. Histoire, Sciences Sociales, 4/2009 (64 année), p. 751-764.

12. Camille Lefebvre, "Itinéraires de sable. Parole, geste et écrit au Soudan central au XIX ${ }^{\mathrm{e}}$ siècle», Annales. Histoire, Sciences Sociales, volume 4, 2009, p. 797-824; Camille Lefebvre, Frontières de sable, frontières de papier. Histoire de territoires et de frontières, du jihad de Sokoto à la colonisation française du Niger, XIX $-X X^{e}$ siècles, Paris, Publications de la Sorbonne, 2015.

13. Hélène Blais, Mirages de la carte. L'invention de l'Algérie coloniale, Paris, Fayard, 2014. 
donne à voir aux Français, ou laisse de côté. La progression du raisonnement cherche à rendre compte des étapes de l'enquête historienne menée à partir de cet unique document qui, analysé pour lui-même puis confronté à d'autres, peut faire sens.

\section{Croquer l'EsPACE : UN DOCUMENT GÉOgRAPHIQUE VIETNAMIEN DRESSÉ À LA HÂTE}

Le croquis étudié ici est, comme toute représentation graphique, un discours adressé dans un but précis et disposant de règles propres ${ }^{14}$. Les signes conventionnels, cette grammaire et ce «langage de la carte ${ }^{15}$, nous montrent d'abord que le document est dressé par un autochtone dans la mesure où s'y retrouvent les caractéristiques principales de la cartographie vietnamienne - il ne peut donc s'agir d'un Français qui connaîtrait les caractères ou le vietnamien romanisé.

Visuellement, le croquis s'organise autour du fleuve, ruban blanc dont les méandres occupent le centre du document. Cela signifie que l'auteur avait une représentation à plat. Celle-ci est courante dans la cartographie vietnamienne, où les cours d'eau occupent généralement l'essentiel de la carte. C'est d'abord le cas pour les géographies royales à partir du XV $\mathrm{XV}^{\mathrm{e}}$ siècle, qui fournissent un tableau précis des provinces et des districts, structurés autour des fleuves ${ }^{16}$. Cela se retrouve aussi sur la représentation d'un segment du fleuve Rouge, non datée, mais effectuée dans un objectif plutôt décoratif : représenté en bleu, le fleuve constitue en effet l'élément central autour duquel s'organisent trois tracés parallèles, en rouge, pour les routes ${ }^{17}$. Le but n'est pas de dessiner le cours réel mais plutôt de montrer qu'il fonde l'organisation spatiale et que les autres composantes s'articulent autour de lui. Si notre croquis a un rôle plus pratique qu'illustratif, il répond bien à cette habitude de placer le fleuve au centre de la carte, de mettre en valeur les embarcadères et les noms des rivières.

Autre composante essentielle de cette cartographie, les montagnes apparaissent ici sous une forme inhabituelle - un figuré en forme de feuille de chêne pour indiquer la masse montagneuse. Elles sont plus souvent symbolisées par la représentation d'une montagne en perspective, voire par une

14. Christian Jacob, L'empire des cartes. Approche théorique de la cartographie à travers l'histoire, Paris, Albin Michel, 1992.

15. François de Dainville, Le langage des géographes. Termes, signes, couleurs des cartes anciennes, 1500-1800, Paris, A. et J. Picard, 1964.

16. Les géographies royales sont établies au bureau des Annales, dépendant du ministère de l'Intérieur, par les historiographes du roi qui compilent les informations rassemblées à travers le pays et dressent les cartes. Sur la dernière géographie royale vietnamienne, probablement commandée par les Français dans les années 1880 afin de disposer d'un tableau du pays par province, voir Ngô Đức Thọ, Philippe Papin et Văn Nguyên Nguyễn [dir.], Đồng Khánh dịa..., op. cit.

17. « [partie du cours moyen du fleuve Rouge (province de Hu $\square$ ng Hóa, huyện de Thanh Thủy et Bảo Thắng)] », Bib. nat. (Bibliothèque nationale de France), Cartes et Plans, GE D 9148. 


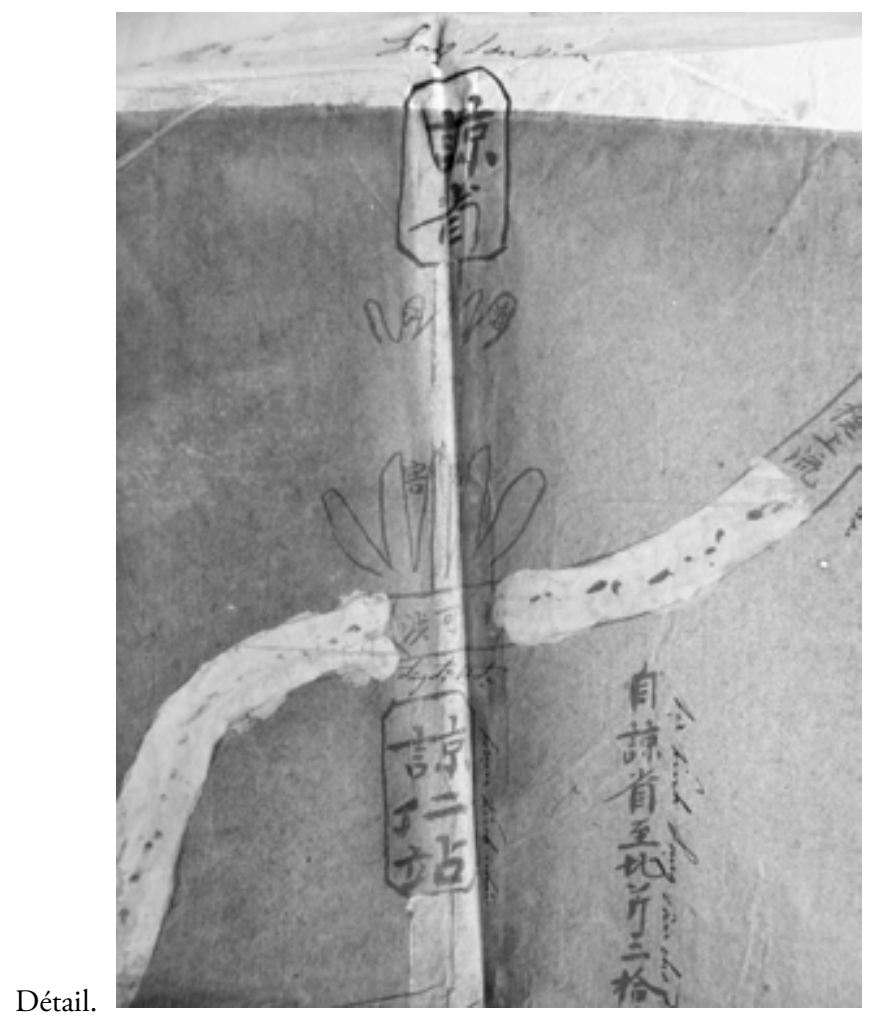

couleur particulière. Sans doute le croquis a-t-il été effectué à la hâte, sans qu'une grande attention soit portée au dessin lui-même ni à l'esthétique du document. Tandis que la végétation apparaît parfois sur les cartes vietnamiennes, seul le relief montagneux est indiqué ici, un texte explicitant le figuré : «succession de montagnes rocheuses", à deux occurrences, ou encore «zone montagneuse». Comme dans la cartographie vietnamienne classique, mais de manière simplifiée, les éléments structurants du paysage sont mis en valeur sur le croquis.

Deux autres aspects sont classiques dans la cartographie vietnamienne : les quatre points cardinaux, donnés par un caractère chinois aux quatre coins de la carte, ainsi que l'absence d'échelle. En revanche, il est plus rare qu'il n'y ait aucune échelle de temps, et donc aucune unité de mesure. La distancetemps, nombre d'heures ou de jours nécessaires pour parcourir la distance entre deux points, est habituellement mentionnée ${ }^{18}$. Cette absence de mesure interroge sur les instruments utilisés, sur les compétences et l'expérience mises en œuvre pour prendre les mesures du terrain, pour en donner une

18. $C f$. par exemple les géographies royales conservées à la Bib. nat., Cartes et Plans, Service hydrographique de la marine, portefeuille 180, division 2. 22. 
représentation conforme à ce qui est attendu. L'absence de ce type d'informations, habituellement fournies, témoigne que le croquis a été dressé à la hâte, par un Vietnamien, avec les matériaux trouvés sur place, ce qui explique ses couleurs et ne l'empêche pas de contenir des renseignements utiles sur le territoire à parcourir.

\section{Dire L'ESPACE : LES ASPECTS PRATIQUES DU TERRITOIRE}

Comme souvent dans la cartographie vietnamienne, le texte pallie les insuffisances du croquis et les deux systèmes sémiologiques cohabitent: image et texte sont en correspondance et s'éclairent mutuellement. Les commentaires textuels fournissent tous des informations pratiques sur le territoire, qui ne sont pas celles habituellement demandées par le pouvoir vietnamien - les géographies royales mentionnent beaucoup de renseignements sur les voies de communication mais jamais avec une telle insistance sur leur état.

Un premier commentaire textuel porte sur la navigabilité du fleuve : «Le fleuve Nhật Đức est actuellement, sous nos yeux, à bas niveau et les petites barques peuvent atteindre l'embarcadère de Trăm». Dans un espace où les cours d'eau constituent des voies de communication primordiales, quand ce ne sont pas les seules, la profondeur, le tirant d'eau, le type d'embarcations qui peut y naviguer ou encore la présence d'obstacles infranchissables conditionnent beaucoup de choses, depuis la bonne marche de l'armée jusqu'aux intérêts commerciaux. Si de nombreuses cartes vietnamiennes présentent des mesures de profondeur et de largeur des rivières, la réponse est ici apportée par le texte.

Après l'enjeu de la navigabilité, une autre note textuelle aborde celui des obstacles au franchissement : «De la province de Lạng Sơn à celle de Bắc Ninh, il y a 36 petits ruisseaux qu'on peut traverser». Cette remarque sur le nombre des cours d'eau à franchir et sur la présence de ponts ou de gués témoigne de nouveau de préoccupations pragmatiques, qui se retrouvent parfois sur d'autres cartes demandées par les Français, où la qualité des ponts - en pierre, en bois - est par exemple notée ${ }^{19}$. Tout est orienté autour du territoire comme espace parcouru ou à parcourir. De nouveau, le texte pallie le caractère incomplet du croquis, sur lequel il est impossible de représenter tous les ponts. Le croquis semble donner les contours du territoire, ensuite décrit plus en détail par le texte.

19. Sur la carte de la "Reconnaissance exécutée par le Né Nguyen Thu Lâm du 18 au 21 septembre [1884]", les ponts sont par exemple nombreux à être figurés et sont accompagnés de précisions quant à leur nom ou leur nature : «Pont en pierre, $4 \mathrm{~m}$ longueur, 0,40 largueur»; SHD, 10 H6, dossier 10. Cartes, levés et croquis, 1884. 


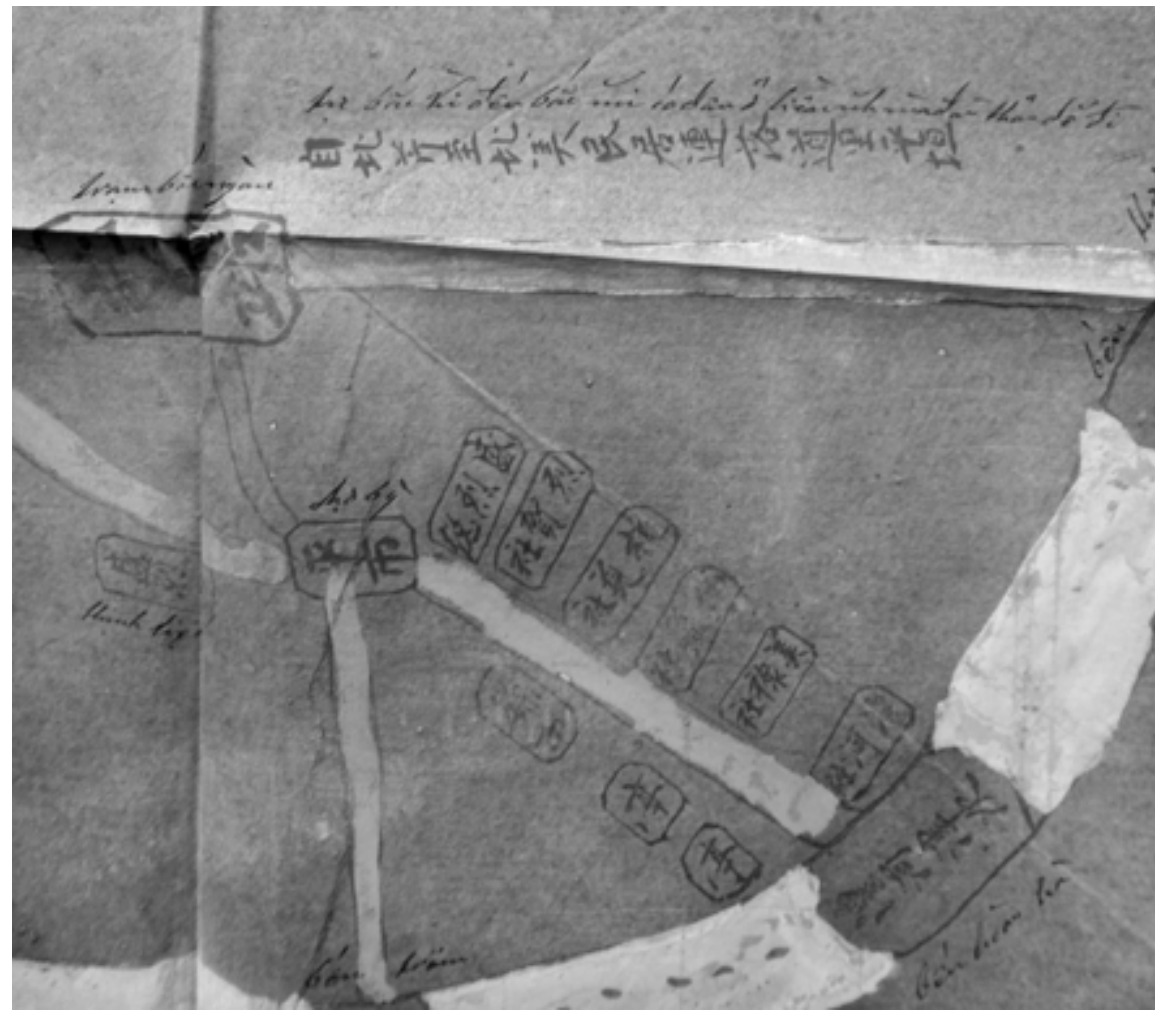

Détail.

Dans l'itinéraire, en général, les différentes étapes comptent aussi afin de pouvoir organiser la marche. C'est ce qui est manifesté sur le croquis par la présence d'embarcadères et de relais qui segmentent le trajet. Les cinq noms, encadrés, écrits en caractères et en écriture vietnamienne romanisée, indiquent les relais de poste qui marquent une jonction entre les deux artères fluviale et terrestre : «trạm Bắc Mĩ, trạm Bắc Ngàn, trạm Bắc Lê, trạm Lạo Quan, trạm Ninh Nhân». Ces derniers se retrouvent généralement sur les cartes françaises de la fin du XIX ${ }^{\mathrm{e}}$ siècle, qui conservent souvent le mot vietnamien "trạm». Aux indications sur l'itinéraire se joignent celles sur les subdivisions administratives de l'espace cartographié, toutes nommées (province, district, préfecture et sous-préfecture). Ce croquis participe en un sens de l'inventaire du monde établi par Claude Nicolet pour l'empire romain ${ }^{20}$. Le but est de donner un état des lieux des axes et des nœuds principaux pour chaque territoire, comme c'est le cas ici.

Les toponymes cessent pourtant à l'échelle villageoise, sans doute parce qu'il est impossible d'inscrire sur le croquis le nom de tous les villages. Cette

20. Claude Nicolet, L'inventaire du monde. Géographie et politique aux origines de l'Empire romain, Paris, Fayard, 1988. 
unité structurante au Tonkin, organisée autour de la maison communale, lieu de réunion et d'accueil, et autour du marché, lieu d'échanges, est cependant bien présente sur le croquis. Le troisième commentaire textuel indique : "Jusqu'au relais de Bắc Mĩ, l'habitat est continu et les routes sont plates». Lorsqu'ils demandent des renseignements aux notables et aux populations locales, les Français posent régulièrement des questions sur la place et le jour des marchés dans les villages, parce que ce sont aussi des lieux de ravitaillement pour leurs ennemis, chinois notamment. Les informations qui figurent sur le document - caractère de l'habitat, relief, relais d'étapes, embarcadères et qualité des routes - permettent donc de penser que ce dernier a été effectué à la demande des Français. C'est en réponse à leurs questions que le document met en exergue l'itinéraire, la route et les étapes, avec des commentaires qui fournissent des points de départ et d'arrivée ainsi que des informations sur l'état de la route. Tous les commentaires vont dans le sens d'une visée utilitaire et inclinent à croire que le croquis s'ajoute à d'autres, également sollicités par les Français dans le cadre de la conquête.

\section{UNE PRISE DE GUERRE?}

On pourra objecter que le croquis aurait pu être simplement trouvé sur le terrain et non commandé par les Français. Il serait alors davantage le butin de la conquête, un trésor de guerre en quelque sorte. Le cas ne serait pas isolé. Il s'est déjà présenté au moment de la conquête de la Cochinchine ${ }^{21}$. Lors de celle du Tonkin également : deux cartes dressées par le major chinois du camp de Long Ping, dans la province du Guangxi, sont trouvées par M. Mauger, pharmacien militaire, et présentées au général Brière de l'Isle - en poste du 8 septembre 1884 au 31 mai 1885, ce qui permet de dater approximativement la découverte ${ }^{22}$. L'une représente le cours de la rivière Noire et du fleuve Rouge, l'autre celui de la rivière Claire - il s'agit donc de représentations graphiques structurées autour des cours d'eau, comme dans le croquis qui nous occupe. À ce moment, aucun interprète chinois n'est présent pour les traduire et elles sont donc rendues à leur propriétaire, M. Mauger. Elles ne sont utilisées que plusieurs mois plus tard, une fois effectuées leur copie, leur traduction et leur comparaison avec les toponymes présents sur les cartes françaises. En mai 1886, alors que le Résident général du Tonkin recherche une carte chinoise de la région de Lào Cai, ces deux documents lui sont proposés par le service des traductions de la résidence. Dans ce cas, il n'est pas précisé si la traduction du chinois a lieu en vietnamien romanisé ou en

21. Hélène Blais, "Cartographie coloniale et savoirs vernaculaires», in Jean-Marc Besse et Gilles Tiberghien [dir.], Opérations cartographiques, Paris, Actes Sud, 2017, p. 206-217.

22. Archives nationales du Vietnam, centre 1, RST 73611, Cabinet du résident général, service des traductions, à M. le Résident général, Hanoi, le 29 mai 1886. 
français - s'il n'y avait que des toponymes, la question importe peu. Sur notre croquis, il est vrai que l'absence de traduction française, notamment pour les commentaires textuels, suscite des questions quant à l'utilisation exacte qui a pu en être faite. L'interrogation témoigne de la tâche difficile pour l'historien d'analyser des cartes souvent séparées des lettres qui les accompagnaient à l'origine. Les croquis tracés par des militaires possèdent souvent le nom de leur auteur, de l'officier responsable de la reconnaissance, une signature, une date, un corps d'appartenance dans l'armée. Dans le cas de croquis dressés par des autochtones, il se peut qu'il y ait une indication au crayon à papier, sur le document ou au dos, sur son auteur ${ }^{23}$ ou à sa provenance ${ }^{24}$, mais ce n'est pas toujours le cas.

Un indice empêche de penser toutefois qu'un Chinois a pu dessiner le croquis, manifestement d'abord écrit en chinois avant d'être traduit en vietnamien romanisé : l'utilisation d'un caractère démotique - le chũ nôm - pour le toponyme «trăm $»^{25}$. Seuls les autochtones utilisent le démotique. Le document n'a donc pas pu être élaboré par un Chinois pour son supérieur mais est bien l'œuvre d'un Vietnamien - n'importe quel notable de village est tout à fait capable de noter ces quelques phrases en caractères chinois. Quant à l'écriture romanisée, elle peut nous renseigner sur le traducteur. Bien que créée au XVII siècle, elle n'est en effet utilisée par les Vietnamiens qu’à partir $\mathrm{du} \mathrm{XX}^{\mathrm{e}}$ siècle et il est donc fort peu probable qu'un mandarin ou fonctionnaire ait pu la connaître en 1883 . Seuls les interprètes attachés aux militaires français, qui venaient souvent du Sud, maîtrisaient le vietnamien romanisé. Ce dernier ne possédait d'ailleurs pas encore de règles fixes, ce qui explique ce qui nous apparait aujourd'hui comme des fautes. Une information, qui n'apparaît qu'en vietnamien romanisé sur la carte, corrobore cette analyse : à côté du district de Hữu Lũng, écrit en caractères et en romanisé, est ajouté en romanisé seulement : «Ici habite le caï [caporal, sergent, garde de l'armée vietnamienne] Vinh». Il est peu probable que l'auteur signe le document : ce n'est pas une pratique courante et il l'aurait fait en caractères chinois. En revanche, il se peut tout à fait que l'interprète vietnamien, après avoir traduit la carte en romanisé, ajoute ce renseignement sur l'auteur du document, nous fournissant par là-même son nom. Les seuls noms d'informateurs dont on dispose apparaissent parfois dans le titre des demandes de renseignements ${ }^{26}$, à moins que son intervention ne soit évoquée dans le rapport militaire joint

23. Au bas de la "Carte de Ninh-Binh " apparaît une signature vietnamienne, "Vô-Lâm-tam-Ky", probablement l'auteur du croquis; Bib. nat., Cartes et Plans, GE D-9070.

24. "trouvé dans la citadelle de Hanoi», Bib. nat., Cartes et Plans, Service hydrographique de la marine, portefeuille 180 , division 2. 22, feuille 4 .

25. Le démotique, ou chĩ nôm, est l'écriture utilisée par les Vietnamiens et basée sur les sinogrammes. Elle sert pour les toponymes locaux. En général, «trăm» s'écrit ainsi : 碟, mais en démotique, comme c'est le cas ici, les deux éléments sont côte à côte et non l'un sur l'autre.

26. " 6 décembre. Un notable de Maï To : $\mathrm{N}^{e}$ Hoang Van Kiem» et «7 décembre. Un notable de Phu Dien, $\mathrm{N}^{e}$ Nguyen Van Dzu", SHD, 10 H6, dossier 15, Rapports des chefs de détachement. Renseignements fournis par les indigènes, 1884-1886. 


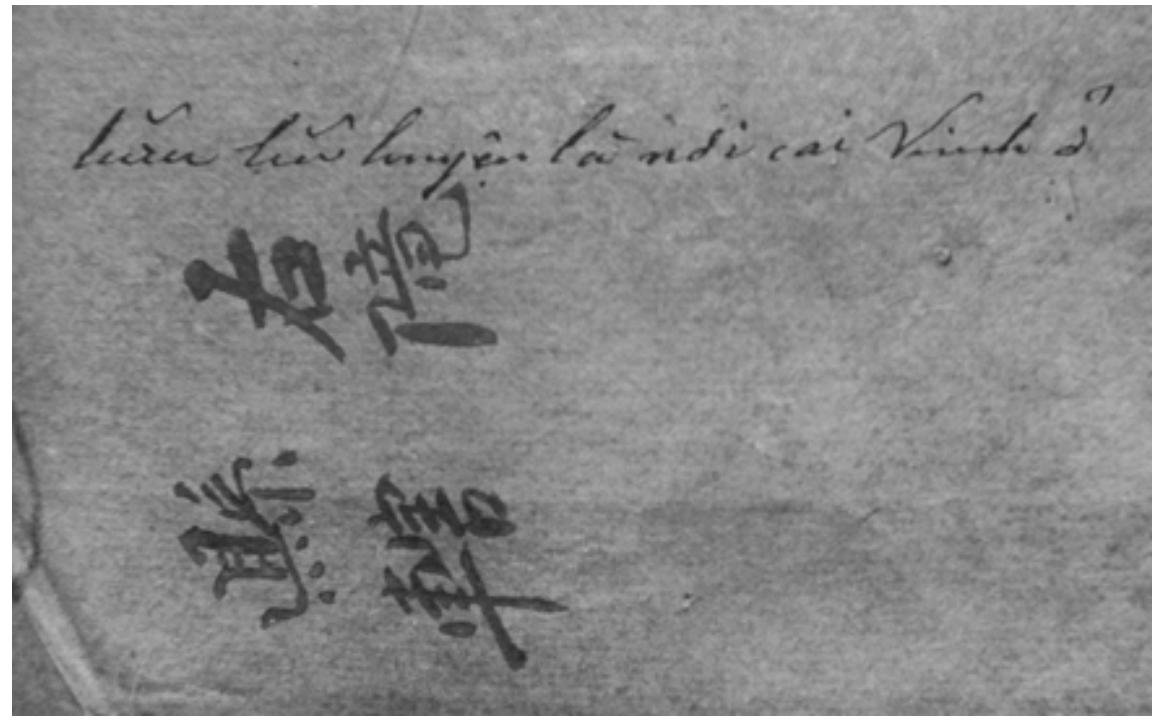

Détail.

au croquis. Et en effet, la mention du caï Vinh sur le croquis nous autorise à faire le lien entre ce document et un rapport de reconnaissance conservé au SHD, qui corrobore l'hypothèse d'une demande faite par les Français.

\section{LE RÉSULTAT D'UNE ENQUÊTE EN SITUATION COLONIALE}

La date de ce croquis, entre 1883 et 1886, appelle quelques remarques sur la cartographie française au moment de la conquête du Tonkin. Les explorations menées jusque dans les régions septentrionales de la péninsule permettent surtout une cartographie d'itinéraires. Lorsque le corps expéditionnaire venu de métropole débarque au Tonkin, les cartes précises manquent et les militaires français doivent composer avec un territoire qu'ils ne connaissent guère. Des consignes sont données pour que, dans chaque colonne militaire, un officier ou sous-officier se renseigne en chemin sur les routes, sur le nom des localités avoisinantes, du district, de la province, et d'autres éléments de cet ordre ${ }^{27}$. Il n'y a guère de précision sur les interlocuteurs à interroger et les archives de la conquête témoignent d'informations apportées par les maires des villages, les notables ou les habitants, quand ce ne sont pas des gens de passage. Le «Voyage d'un marchand de poisson de Luc-Ngan à Langson", recueilli dans cette ville en 1884, donne ainsi à voir un véritable formulaire de demande de renseignements à travers la succession

27. SHD, $10 \mathrm{H} 15$, dossier 3 , sous dossier 4, Instructions pour faire suite à l'ordre $\mathrm{n}^{\circ} 8$, du général Brissaud. 
des questions posées au marchand et des réponses données ${ }^{28}$. Les préoccupations des topographes français sur la longueur des routes, les étapes et la présence de ponts apparaissent clairement. Cette enquête orale donne parfois lieu à une représentation graphique, soit que l'officier français trace un croquis à partir des informations recueillies, soit qu'un de ses interlocuteurs lui en fournisse une, comme cette "carte dressée et apportée par le maire de Yen Chau ${ }^{29}$, en 1885 au commandant du poste de Núi Bóp. Dès lors, conservé dans ces mêmes archives de la conquête et présentant tous les éléments dignes d'intérêt pour les Français, le croquis soumis à notre étude est probablement le produit d'une telle enquête.

Un texte conservé dans un autre dossier permet d'envisager des éléments précis à ce sujet. En effet, le 7 juin 1884, au terme d'une expédition de quelques jours en chaloupe sur le Sông Thương, au départ de Phủ Lạng Thương (province de Bắc Ninh), l'officier Durand écrit : "Je suis monté au village de Dao-Quan, où j'ai pris des renseignements près du chef de canton, qui m’a mis en relation avec un ancien caï de l'armée régulière annamite qui connaît très bien la route ${ }^{30}$. Si le gradé vietnamien n'est pas nommé, sa mention, la similitude des lieux et la concordance de la date sont autant d'éléments qui autorisent un rapprochement. La lettre se poursuit : "Il ressort de ces renseignements que la route et le Song Thuong se côtoient à hauteur du village de Voï-Xo, limite extrême de la première reconnaissance du petit Léopard. Interrogé par moi, le caï répondit que Voï-Xo est situé à environ 3 heures de marche de Kep, ce qui est à peu près exact». Cela montre d'une part l'intérêt effectif de l'officier français pour les voies de communication, puisque la quête de renseignements porte d'abord sur le fleuve et la route. D'autre part, la question de la distance-temps apparaît bien comme une composante du dialogue entre les deux militaires - et un moyen pour le Français de vérifier les compétences de son interlocuteur. Ce sont ces éléments qui se retrouvent sur le croquis dressé par les membres de l'expédition : le fleuve est représenté au centre, avec des mesures, et la distance en jours de marche est donnée à deux reprises. Peut-être ce croquis a-t-il pu servir de modèle au caï Vinh : le Vietnamien a-t-il établi son croquis à partir de sa simple représentation spatiale mentale ou a-t-il puisé à d'autres sources? Il reste cependant malaisé de faire la part entre ce qui, dans son croquis, est hermétique à une influence étrangère, ce qui ressort de l'adaptation et ce qui

28. «Combien de jours de marche? 2 jours et $1 / 2$ - pas marché la nuit. Dans quelle saison? Le $12^{\mathrm{e}}$ mois $1^{\mathrm{re}}$ couchée - où ? parti de bonne heure. On arrive le soir à Cho Nuu (ou Cho Chu). Terrain plat [...] jusqu’à Cho Chu $1 \mathrm{~m}$ de largeur environ; de Cho Chu à Langson 1,80 m environ. Ponts? [...] ruisseaux en ont, d'autres pas. Rivières? plusieurs ruisseaux de 4 à $5 \mathrm{~m}$. Pas de grande rivière.», SHD, $10 \mathrm{H6}$, dossier 13, renseignements sur le Loch-Nam et les routes de Lang-son. Rapports de la place (Bac-Ninh), 1884-1885.

29. SHD, 10 H6, dossier 16, "Noui Bop, le 12 mars 1885. Le capitaine Falcon commandant le poste de Noui Bop à Monsieur le Colonel Commandant la circonscription Phù".

30. SHD, $10 \mathrm{H6}$, dossier $\mathrm{n}^{\circ} 14$, lettre de Durand, Phu Lang Thuong, à M. le lieutenant colonel commandant supérieur à Bac Ninh, 7 juin 1884. 
est de l'ordre de la réplique - à partir du croquis français qui aurait pu servir de modèle dans le choix des figurés ${ }^{31}$. Un dernier élément permet de mettre en rapport de manière évidente le texte et le croquis vietnamien : l'officier français évoque la reconnaissance antérieure de la chaloupe et écrit qu' «à ce moment, il y avait une crue, qui n'existe plus, et une chaloupe avec les eaux aussi basses ne passerait pas, mais les jonques peuvent remonter jusque-là». Ce renseignement corrobore celui fourni par le caï Vinh sur le croquis à propos du bas niveau de l'eau et de la navigabilité limitée aux seules barques - ou aux jonques, selon l'officier français. Il manque certes l'ultime information qui permettrait de confirmer de manière certaine toutes ces hypothèses : nulle part, l'officier français ne dit avoir demandé une carte au caï qu'il a interrogé. Il reste donc une infime inconnue sur le lien entre enquête orale, rapport écrit et document cartographique. Tout converge cependant vers la conclusion que le croquis aurait été dressé par le caï Vinh à la demande des Français, traduit par un interprète vietnamien venu du Sud et envoyé ensuite aux archives françaises après la conquête, après avoir servi sur le terrain.

*

Muet à bien des égards et pourtant riche de renseignements, le document présenté ici fait partie des représentations spatiales dressées à l'échelle locale, par des notables vietnamiens, à la demande des Français. L'absence d'une telle cartographie avant la période coloniale, la présence du croquis dans les archives françaises de la conquête du Tonkin (1883-1886), les indications utiles qu'il contient et qui apparaissent comme autant de réponses à une sollicitation extérieure, l'utilisation de caractères chinois, du démotique, et la transcription en vietnamien romanisé, les éclairages apportés par le rapport de reconnaissance, enfin, fournissent des arguments pour confirmer l'hypothèse initiale. L'analyse ne pourra cependant jamais rendre l'entière vérité de ce croquis : l'intention de l'auteur, son degré de liberté dans la confection de la carte, dans le choix des éléments à représenter et la raison d'une absence de traduction en français, restent inconnus. En ce moment charnière où tout peut basculer, puisque les Français ne sont pas solidement implantés au Tonkin, leur demande de renseignements géographiques est compréhensible mais la réponse du Vietnamien reste plus mystérieuse.

Résultat d'une enquête, d'une rencontre en situation coloniale, en situation de conquête aussi, ce croquis montre que le savoir autochtone doit être suscité, qu'il est le résultat d'un besoin exogène qui s'exprime mais n'existe pas en soi $^{32}$. C'est parce qu'un Français devait pouvoir se repérer que son

31. Philippe Papin, «Un laboratoire pour l'étude des transferts», in Hoai Huong Aubert-Nguyen et Michel Espagne [dir.], Le Vietnam. Une histoire de transferts culturels, Paris, Demopolis, 2015, p. $33-46$.

32. C'est aussi le cas sans l'intervention des Français : c'est parce que le voisin veut racheter la 
intervention, extérieure, a suscité le dialogue et in fine la production d'un document cartographique autochtone. Le document nous interpelle alors sur une mise en commensurabilité des savoirs. Si les nombreuses inconnues qui persistent ne permettent pas une histoire à parts égales ${ }^{33}$ à travers les cartes, cette représentation autochtone offre toutefois un contrepoint enrichissant dans la lecture des archives coloniales françaises ${ }^{34}$. Elle justifie que l'on s'y intéresse, sans lui donner une place disproportionnée qui n'était pas la sienne. Les documents disponibles sont riches mais peu nombreux et leur intérêt ne doit pas masquer leur rareté. Il s'agit de traces éparpillées dont la force d'attraction est renforcée par leur caractère exceptionnel; traquées dans les archives, érigées au rang de découverte, elles n'en restent pas moins souvent silencieuses à maints égards. Leur principal intérêt est de témoigner de la rencontre en situation coloniale et de montrer qu'au-delà des clivages entre savoirs traditionnels et sciences modernes, Vietnamiens et Français se retrouvent autour d'une représentation du territoire comme espace parcouru et administré.

Marie de Rugy est post-doctorante à l'Université de Cambridge, faculté d'histoire-Wolfson College

terre qu'il faut apprendre à la mesurer, parce qu'un mandarin exige des comptes qu'il faut apprendre la comptabilité.

33. Romain Bertrand, L'histoire à parts égales. Récits d'une rencontre Orient-Occident (XVIe-XVII siècle), Paris, Seuil, 2011.

34. Ann Laura Stoler, Along the Archival Grain. Epistemic Anxieties and Colonial Common Sense, Princeton, Princeton University Press, 2009. 